network; and 3) Impact of physical health on and from dementia. Our findings explore the dynamics of trust when establishing the limitations of physical health treatments in people with dementia. We found that prescribed treatments were unhelpful when they were not adapted to the context of dementia and where strategies depended on memory. Family carers at times appeared to adopt an 'all or nothing' approach to physical health decision making due to the lack of flexible interventions. This resulted in the voice of people with dementia being lost when determining the benefits of treatment. Treatment was, at times, continued with questionable benefit, or even against the preferences of the person with dementia. A successful balance between comfort and quality of life were achieved when approaches were discussed and agreed in collaboration with all stakeholders.

Conclusion Care decisions must consider people with dementia as a whole, and be based on realistic outcomes, best interests and collaboratively agreed preferences.

\section{ENACTING TRUST WHEN RECRUITING PEOPLE WITH AN INTELLECTUAL DISABILITY INTO HEALTH RESEARCH}

\section{A Russell. University of Leeds, UK}

10.1136/bmjopen-2021-QHRN.29

Research regulation frames people with learning disabilities as a 'vulnerable' group. Trust may be too readily given, it is assumed. The authority of the recruiter is assumed when recruiting in clinical settings. In my research I will explore how trust operates in a series of recruitment experiences and critique simplistic understandings of trust that partner assumptions about a participant's capacity and autonomy.

Often research recruitment is conceptualised as a dyadic relationship between researcher and subject of research with the researcher often seen as a figure of power and authority. Inter-personal interactions and relational autonomy all play their part in research recruitment and require interrogation if we are to move beyond a value free concept of 'gold standard' research and see the labour of the individuals behind the findings.

Methods I will explore the operation of trust through an autoethnographic analysis of journal entries made by me during my recruitment to an applied health study for people with a learning disability.

This contested method has been selected to give a voice to the often unarticulated decisions made during recruitment of participants deemed 'vulnerable.' It exposes the interplay of power and trust, examining the multiple layers of ambition, guilt, embarrassment and confusion not readily accessible in interviews or observation (Throsby 2018). I will assess how this researcher operationalizes trust-gaining behaviour as part of recruitment and question the ethics of this performance.

Results and conclusions I explore ideas of autonomy, vulnerability and trust in relation to research recruitment, situating the findings in the context of health research from the researcher perspective. I will examine 'those particular trustwarranting properties and their signs' (Hedgecoe 2012) and how researchers enact these signs to meet their objectives, while questioning the ethics of enacting trust and the labour of the researcher when trying to meet these multiple expectations.

\section{WHAT IMBIBES TRUST IN CHATBOT USERS? A MULTIDIMENSIONAL VIEW IN CONTEXT TO HEALTHCARE SERVICES DURING PANDEMIC}

S Saxena, A Ahmed, Amritesh. Indian Institute of Technology, Ropar Punjab, India

\subsection{6/bmjopen-2021-QHRN.30}

Introduction Smart technology, like AI-enabled chatbots, has transformed how healthcare consumers (patients) co-create value with their service providers (doctors). However, for effective co-creation, consumers also have to equally integrate their resources (like information). Here, trust plays an important role. If consumers do not trust the chatbots, they will contribute fewer resources resulting in value co-destruction. This dynamic of trust and resource integration is further challenged in uncertain times (like COVID-19). Thus, it becomes imperative to explore the factors that imbibes/ abstain trust within chatbot users while consuming healthcare services during a pandemic? This study focuses on this question. Additionally, since trust has two sides (cognitive $\&$ affective) and the factors affecting them can act from two different levels of service ecosystem (individual \& institutional); therefore the study categorizes the trust factors using a $2 \times 2$ matrix, i.e., (Individual $\&$ cognitive, Institutional \& cognitive, Individual \& affective, Institutional \& affective). There is hardly any study that adopts this multifaceted view of trust factors in AI-empowered healthcare services.

Method The study uses an inductive qualitative approach, where thirty regular chatbot users were interviewed using a semi-structured interview. Excerpts from the interviews were thematically coded and analyzed (using QSR Nvivo-12). Identified factors are validated using social simulation approach.

Results The study identifies 16 key factors influencing consumers' trust in chatbot based on $2 \times 2$ matrix proposed in this study. To cite a few of them, Cognitive trust factors: perceived knowledge, level of task orientation in interaction, level of automation; affective trust factors: avatar like presence, level of emotional support offered, perceived honesty \& ethics, perceived security \& privacy.

Conclusion The results show that consumers phenomenologically process the trust in chatbot usage getting influenced both cognitively and emotionally at multiple levels. Thus, practitioners should accordingly plan the 'trust-building strategy'.

\section{GENDER AND SEXUALITY DIVERSE (GSD) WOMEN'S EXPERIENCES OF CANCER AND CANCER CARE}

S Sperring, K Allison, R Power, C Ellis, J Ussher, J Perz, A Hawkey. Translational Health Research Institute, Western Sydney University, Australia

\subsection{6/bmjopen-2021-QHRN.31}

Background This paper will present the preliminary findings of research into gender and sexuality diverse (GSD) women's experiences of cancer and cancer care, as part of a broader ARC funded project titled 'Out with Cancer'. LGBTQI+ communities experience a disproportionate cancer burden, and face unique psychosocial challenges, such as higher rates of cancer related distress and sexual concerns, lower levels of family support, difficulties in accessing general health care or cancer services, gaps in patient-provider communication and lower satisfaction with cancer care. 